\title{
Nozzle condition monitoring in 3D printing
}

\author{
Yedige Tlegenov, Geok Soon Hong*, Wen Feng Lu \\ Department of Mechanical Engineering, National University of Singapore, \\ 9 Engineering Drive 1, Singapore 117575
}

\begin{abstract}
3D printing and particularly fused filament fabrication is widely used for prototyping and fabricating low-cost customized parts. However, current fused filament fabrication 3D printers have limited nozzle condition monitoring techniques to minimize nozzle clogging errors. Nozzle clogging is one of the most significant process errors in current fused filament fabrication 3D printers, and it affects the quality of the prototyped parts in terms of geometry tolerance, surface roughness, and mechanical properties. This paper proposes a nozzle condition monitoring technique in fused filament fabrication 3D printing using a vibration sensor, which is briefly described as follows. First, a bar mount that supports the liquefier in fused filament fabrication extruder was modeled as a beam excited by a system of process forces. The boundary conditions were identified, and the applied forces were analyzed for Direct and Bowden types of fused filament fabrication extruders. Second, a new 3D printer with a fixed extruder and a moving platform was designed and built for conducting nozzle condition monitoring experiments. Third, nozzle clogging was simulated by reducing the nozzle extrusion temperature, which caused partial solidification of the filament around inner walls of the nozzle. Fourth, sets of experiments

\footnotetext{
* Corresponding author

Email address: mpehgs@nus.edu.sg (Geok Soon Hong)

This is a pre-print of an article Yedige Tlegenov, Geok Soon Hong, Wen Feng Lu, Nozzle condition monitoring in 3D printing, Robotics and ComputerIntegrated Manufacturing, Volume 54, December 2018, Pages 45-55, ISSN 0736-5845, at: https://www.sciencedirect.com/science/article/pii/S073658451730443X
} https://doi.org/10.1016/j.rcim.2018.05.010. The final authenticated version is available online
\end{abstract}


were performed by measuring the vibrations of a bar mount during extrusion of polylactic acid, acrylonitrile butadiene styrene, and SemiFlex filaments via Direct and Bowden types of fused filament fabrication extruders. Findings of the current study show that nozzle clogging in fused filament fabrication 3D printers can be monitored using an accelerometer sensor by measuring extruder's bar mount vibrations. The proposed technique can be used efficiently for monitoring nozzle clogging in fused filament fabrication 3D printers as it is based on the fundamental process modeling.

Keywords: 3D printing, Fused Deposition Modeling, Fused Filament Fabrication, Condition Monitoring, Nozzle Clogging

\section{Introduction}

Additive manufacturing (AM) is the process of joining materials layer by layer to build 3D objects [1]. One of the most widely used AM processes is fused deposition modeling (FDM) patented by Stratasys [2]. After one of the first

5 FDM patent was expired in 2009, a generic term called fused filament fabrication (FFF) was introduced, allowing 3D printers to self-replicate and develop further [3]. This lead to the development of the RepRap project utilizing open-source 3D printer hardware and software [4], which drastically decreased the cost of FFF 3D printers. The development of open-source RepRap project brought the 3D printing technology to a wide range of users [5]. Therefore, the applications of FFF 3D printers have been introduced in various fields, namely, education [6], [7], rapid prototyping [8], [9], robotics [10], [11], scientific tools [12], [13], and tissue engineering [14], [15].

The FFF machine fabricates 3D parts that are first modeled using computer15 aided design (CAD) software, then converted into a STereoLithography format (.stl) with surface geometry parameters. During the FFF process, a filament material is fed into the heater block where it melts and extrudes onto a build platform via controlled three axis stage. This forms a thin cross-sectional layer of a part, and the process repeats by forming all cross-sectional layers until the 
part is fully fabricated. FFF filaments are commonly made of thermoplastics, for example, polylactic acid (PLA), acrylonitrile butadiene styrene (ABS), SemiFlex, and others. The applications of the FFF technology have been explored for manufacturing low-cost detailed parts, prototypes, and customized manufacturing tools. However, there are numbers of quality problems of the parts fabricated using FFF process, and according to previous research, there is a $20 \%$ failure rate for inexperienced users of FFF 3D printers [5]. For example, Wang et al. [16] studied that various process parameters can influence the surface finish of $3 \mathrm{D}$ printed parts, and Boschetto et al. [17] showed that poor surface roughness might lead to the need for secondary finishing operations. Properties of parts fabricated by FFF were characterized by Ahn et al. [18], and they showed that FFF 3D printed parts had mechanical strength issues in comparison with injection-molded parts. Moreover, Tymrak et al. [19] investigated mechanical properties of components $3 \mathrm{D}$ printed with ABS and PLA materials under realistic environmental conditions. They showed that extrusion temperature could have a significant impact on the mechanical properties of 3D printed parts. A heat conduction model for predicting the adhesion quality between filaments was developed Costa et al. [20]. Their results showed that the environment temperature affected filament adhesion thus influenced the mechanical properties of FFF processed parts. Kaveh et al. [21] analyzed the effect of extruded temperature, flow rate, 40 feed rate, raster angle, and raster width on the accuracy of FFF prototyped parts and showed that extruded temperature and raster width have the most significant effect among other process parameters. A temperature-control 3D printing system was introduced by Yang et al. [22] to investigate the effects of the nozzle temperature, ambient temperature, and heat treatment methods on the mechanical properties of FFF 3D printed prototypes. Their results showed that tensile strength has a direct relationship with nozzle temperature and ambient temperature in FFF.

The above-mentioned quality problems of parts fabricated by FFF can be influenced by nozzle clogging conditions that may vary during fabrication. Nozzle clogging can cause geometrical misalignments or may lead to failure during 3D 
printing [23], and thus can be considered as one of the critical process errors. Presently, the machine operator detects the clogging, stops the print, manually cleans the nozzle, and fabricates the part again from the beginning. Hence, there is a need in developing nozzle condition monitoring technique in FFF, and it can be very promising for a better quality of the prototyped parts in terms of surface roughness and mechanical properties. In summary, FFF is one of the most popular and widely explored AM processes but has a number of quality problems of fabricated parts due to nozzle clogging. Thus, one of the solutions is to develop nozzle condition monitoring technique in FFF 3D printing.

Several studies have been reported in the area of monitoring in extrusion-based AM processes. A multi-camera system and image processing software was used by Straub [24] to detect completion failure defects and print quality. Later, he used a multi-camera visible light 3D scanning system to detect interior 3D print errors and obscured exterior surfaces [25]. Augmented Reality (AR) toolkit was used by

${ }_{65}$ Ceruti et al. [26] to track the geometry of 3D printed part real-time and compare with the ideal CAD model using AR glasses. Furthermore, computer vision based low-cost real-time FFF monitoring technique was proposed by Nuchitprasitchai et al. [27], [28]. They introduced an algorithm for reconstructing 3D images from overlapping 2D intensity measurements using low-cost web cameras around the 3D printed part. Their results showed that single and double camera system algorithms were efficient at monitoring a clogged extruder, failed print, or filament absence. Greeff and Schilling [29] introduced a filament slippage measurement technique in fused deposition modeling, where extrusion volumetric flow rate was monitored real-time and by closing the loop of the extruder they reduced the filament slippage amount. Holzmond and Li [30] proposed a quality monitoring method in 3D printing to track the geometry of the part during fabrication while comparing actual geometry data with the CAD model, which allowed to estimate the dimensional errors in situ. Fang et al. [31] presented vision-based monitoring in layered AM, where the optical image of each layer have been so compared with the ideal layers from the CAD model during fabrication. Faes et al. [32] developed a method of profile monitoring during 3D printing via $2 \mathrm{D}$ laser 
triangulation scanner and analyzed feedback signals. Rao et al. [33] proposed online quality monitoring in the fused filament fabrication process using multiple sensors, such as accelerometers, thermocouples, video borescope, and an infrared temperature sensor. After conducting sets of experiments, they presented the empirical relationships between input parameters (feed/flow rate ratio, layer height, and extruder temperature) and surface roughness of the prototyped part. Wu et al. [34] proposed in-situ monitoring of FFF machine condition via acoustic emission technique. They used time-domain features of acoustic emission data to identify experimentally several abnormal states of a 3D printer, namely, run out of material, semi-blocked extruder, and completely blocked extruder. Baumann et al. [35] designed and implemented a sensor array for desktop 3D printers, consisting of motion, orientation, vibration, temperature, and hygrometry sensors. After running number of experiments, the printing and not-printing states were empirically distinguished. Despite the fact that the studies mentioned above have proposed various monitoring techniques for layered AM processes using different sensors, they were not based on the process dynamics model, and thus had some limitations to fully interpret or predict the process errors. Bukkapatnam and Clark [36] reported a physics-based dynamic model of contour crafting layered AM process. They modeled the AM machine as a dynamic lumped mass system excited by filament feeding force, drive mechanism force, and others. Furthermore, they measured the vibrations gathered from accelerometers placed on the extruder head and the machine frame. From the vibration signals data, they identified several process errors, namely, over/underflow, fast/slow feed. They captured more severe vibrations of the AM machine and therefore their dynamic model paid less attention to the the back-pressure force. To sum up, there are numbers of monitoring techniques reported for layered AM processes. Despite the above-mentioned condition monitoring approaches developed for AM processes, perhaps no studies have been carried out to monitor precise nozzle clogging conditions in FFF 3D printing.

The objectives of the current study are to a) propose a physics-based model that represents the FFF process with regards to nozzle condition, particularly, 
clogging; b) propose a nozzle condition monitoring method in FFF using a vibration sensor. The novelty of the current method is modeling of FFF extruder's bar mount as a beam and measuring its vibrations resulting from the blocked nozzle.

Following the Introduction, Section 2 first introduces the theoretical model of FFF process with regards to nozzle condition; and describes theoretical and experimental methods of simulating nozzle clogging that can be detected via vibration sensor. Section 3 presents the results of theoretical and experimental simulation of nozzle clogging. Section 4 evaluates the results and discusses the importance of the nozzle condition modeling and monitoring technique using an accelerometer. Section 5 summarizes and concludes the proposed study.

\section{Modeling of FFF Process}

The methodology in the following section includes modeling of FFF process with regards to nozzle clogging, preparation for nozzle condition monitoring experiment, simulation of the clogged nozzle, and the experimental procedure.

\subsection{Physics-based theoretical modeling of FFF process}

There are two main types of FFF extruders, namely, Direct extruder and Bowden extruder as shown in Figure 1 and Figure 2, respectively. The difference between Direct and Bowden extruder is the distance from the motor drive gear to the heated liquefier. The Direct extruder's motor is mounted right on the top of the heated liquefier, while the Bowden's motor is placed away. The heated liquefier in both extruders is connected to the bar mount from the top side. The FFF process modeling methodology consists of a problem statement, identification of applied loads, and beam analysis.

\subsubsection{Problem statement}

As can be seen from Figure 1 and Figure 2, the nozzle together with the liquefier is connected to the bar mount from the top for both types of extruders.

Therefore, it can be noted that any nozzle disturbances along the z-axis are also 
affecting the contact area of the liquefier with the bar mount from the top. Thus, current study proposes to track the changes of the nozzle condition during 3D printing by measuring the vibrations of the bar mount along the z-axis. For this reason, the bar mount for both extruder types was modeled as a beam with appropriate boundary conditions and material properties. Furthermore, any forces affecting the nozzle along the z-axis were modeled to be distributed over a contact area of the heated liquefier with the bar mount from the top. Moreover, the loading applied to the nozzle-liquefier-bar mount was modeled as the system of two main opposite forces. The proposed modeling layout for Direct and Bowden extruder are shown in Figure 3 and Figure 4 respectively. To summarize, the physics-based model proposed in the present study was based on the beam analysis and can be applied for the most widely used types of FFF extruders, such as Direct and Bowden extruders.

\subsubsection{Identification of applied loads}

155

The system of applied loads acting on the FFF extruder mainly consists of feed force and back-pressure force. The feed force is a function of motor torque, drive gear parameters, filament properties, and the distance between two locations where the filament feeds and where the filament enters into the liquefier (also called as unsupported filament length). The back-pressure force is a function of the pressure drop inside of the heated liquefier and the nozzle. The vector summation of these two forces was modeled as a partially distributed uniform load acting on a bar mount beam.

Filament feed force pushes the filament to the liquefier. Assuming the system with two feeding rollers, the filament feed force for both Direct and Bowden extruder types can be written as [37]

$$
F_{\text {feed }}=\frac{2 T_{z}}{R_{\text {rol }}},
$$

where $T_{z}$ is filament feeding stepper motor torque, $R_{\text {rol }}$ is filament feeding roller radius. However, the Direct extruder has the upper limit that the feeding motor can provide. This is due to the fact that the filament in Direct extruder has 
unsupported region where compression can reach a critical limit, which may lead to filament buckling or breakage. The critical force that the feeding motor can place on the filament in the Direct extruder derived from an Euler buckling analysis for pin-ended boundary condition [38]

$$
F_{f e e d C R}=\frac{\pi^{2} E_{f i l} I_{f i l}}{L_{u l f}^{2}},
$$

where $F_{f e e d C R}$ is critical feed force, $E_{f i l}$ is Young's modulus of the filament, $I_{f i l}$ area moment of inertia of the cross section of the filament, $L_{u l f}$ is filament unsupported length.

Back-pressure force for both types of extruder depends on the pressure drop and acts in opposite direction to the filament feed force inside of the liquefier where filament melts and extrudes through the nozzle. The isothermal pressure drop inside of the nozzle can be derived as the sum of all pressure drops in three nozzle zones [39]:

$$
\begin{gathered}
\Delta p_{1}=2 L_{1}\left(\frac{v}{\chi}\right)^{\frac{1}{q}} \cdot\left(\frac{q+3}{R_{1}^{q+1}}\right)^{\frac{1}{q}} \\
\Delta p_{2}=\frac{2 q}{3 \tan \left(\frac{\alpha}{2}\right)} \cdot\left(\frac{1}{R_{1}^{\frac{3}{q}}}+\frac{1}{R_{2}^{\frac{3}{q}}}\right) \cdot\left(\frac{v R_{2}^{2}(q+3)}{\chi}\right)^{\frac{1}{q}}, \\
\Delta p_{3}=2 L_{3}\left(\frac{v}{\chi}\right)^{\frac{1}{q}} \cdot\left(\frac{(q+3) R_{1}^{2}}{R_{2}^{q+1}}\right)^{\frac{1}{q}},
\end{gathered}
$$

where $\Delta p$ is isothermal pressure drop inside of the nozzle, $v$ is flow mean velocity, $q$ is flow behavior index, $\chi$ is flow consistency index $L_{1}, L_{3}$ are nozzle length values in the zones 1 and 3 respectively, $R_{1}, R_{2}$ are nozzle radius values at the entry and at the outer regions respectively, is an inside angle of the nozzle.

These three nozzle zones are illustrated in Figure 5. Thus, the total isothermal pressure drop inside of the nozzle is calculated [39] as

$$
\Delta p=\Delta p_{1}+\Delta p_{2}+\Delta p_{3}
$$


Consequently, in order to obtain the temperature dependent pressure drop, the Arrhenius Law can be applied [40]

$$
H(T)=e^{\beta\left(\frac{1}{T}-\frac{1}{T_{0}}\right)}
$$

where $T$ is extruding temperature and $T_{0}$ is reference temperature. Hence temperature dependent pressure drop can be obtained by [40]

$$
\Delta p_{T}=\Delta p \cdot e^{\beta\left(\frac{1}{T}-\frac{1}{T_{0}}\right)} .
$$

Therefore, the back-pressure force for Direct and Bowden extruder types can be derived as

$$
F_{b p}=\Delta p_{T} \cdot A_{f i l},
$$

where $A_{f i l}$ is filament's cross sectional area.

After calculating feed force and back-pressure force, the total force acting on 195 a beam can be written as

$$
W=F_{\text {feed }}-F_{b p},
$$

where $W$ is a total uniform force acting on a simply supported beam over a partial length.

\subsubsection{Beam analysis}

The bar mount for Direct and Bowden types of extruders was modeled as a beam with known material properties and geometrical parameters, as shown in Figure 3 and Figure 4. As can be seen from Figure 3 and Figure 4 for Direct and Bowden extruder types respectively, the bar mount can be analyzed as a simply supported or pinned-pinned beam with a partially distributed uniform loading as shown in Figure 6. Assuming the Euler-Bernoulli beam to have uniform material properties and a uniform cross section, the governing equation of the 
beam model can be written as [41]

$$
m(x) \ddot{z}(x, t)+c(x) \dot{z}(x, t)+E I \frac{\partial^{4} z(x, t)}{\partial x^{4}}=f(x, t),
$$

where $m(x)$ is beam mass per unit length, $c(x)$ is beam viscous damping coefficient per unit length, $E$ is Young's modulus of the beam, $I$ is cross sectional second moment of area of the beam, $z(x, t)$ is lateral displacement of the beam, $f(x, t)$ is force function acting on the beam. Thus, the modal solution for the lateral displacement of the beam $z(x, t)$ can be written as

$$
z(x, t)=\sum_{j} \phi_{j}(x) q_{j}(t), \text { for } j=1,2, \ldots
$$

where $\phi_{j}(x)$ is $j$-th normal mode of the system, $q_{j}(t)$ is generalized coordinate. Hence, there is a need in calculating the mode shape by taking into consideration the modal solution [41]

$$
\phi_{j}(x)=C \cos \lambda_{j}+D \sin \lambda_{j}+E \cosh \lambda_{j} x+F \sinh \lambda_{j} x,
$$

where $C, D, E, F$ are boundary condition constants, $\lambda$ is an argument of characteristic equation of the beam. In present study, the bar mount was modeled as a pinned-pinned beam, thus the deflection and the moment at the analyzed beam ends are equal to zero. The boundary conditions for the pinned-pinned beam can be defined as

$$
\begin{aligned}
& \text { for } x=0 ; \quad x(0, t)=0 ; \quad E I \frac{\partial^{2} z(0, t)}{\partial x^{2}} \text {; } \\
& \text { for } x=l ; \quad x(l, t)=0 ; \quad E I \frac{\partial^{2} z(l, t)}{\partial x^{2}} .
\end{aligned}
$$

220 After applying the boundary conditions (14), (15) into equation of mode shapes (13), it is possible to derive [42]

$$
\phi_{j}=\sqrt{2} \sin \lambda_{j} x=\sqrt{2} \sin \frac{j \pi x}{l},
$$


where $l$ is length of the beam. The amplitude multiplication to $\sqrt{2}$ was done for simplicity in solving the generalized mass. Thus, the resonant frequencies can be defined as

$$
\left(\lambda_{j} l\right)^{4}=\frac{m l^{4}}{E I} \omega_{j}^{2},
$$

where $\omega_{j}$ is modal frequency, $m$ is mass of the beam, $\lambda_{j} l=j \pi$, for $j=1,2, \ldots$. After calculating the generalized mass and the generalized force, as elaborated in [42], the generalized coordinate can be obtained as

$$
\begin{aligned}
& q_{j}(t)=\left(\frac{2 \sqrt{2} W}{\lambda_{j} m l}\left(\sin (b+a) \frac{\lambda_{j}}{2} \cdot \sin (b-a) \frac{\lambda_{j}}{2}\right) \sin \omega t\right) . \\
&\left(\frac{1}{\omega_{j}^{2}-\omega^{2}+i 2 \zeta_{j} \omega_{j} \omega}\right),
\end{aligned}
$$

where $\zeta_{j}$ is damping ratio. Consequently, the mode shape from (16) and the generalized coordinate from (18) can be substituted into (12). Therefore, the general form of the lateral displacement of pinned-pinned beam excited by uniform loading distributed over a partial length can be obtained as [41]

$$
\begin{aligned}
z(x, t)=\frac{4 W}{m l} \sum_{j}^{\infty} \frac{\sin \lambda_{j} x}{\lambda_{j}\left(\omega_{j}^{2}-\omega^{2}+i 2 \zeta_{j} \omega_{j} \omega\right)} . & \\
& \left(\left(\sin (b+a) \frac{\lambda_{j}}{2} \cdot \sin (b-a) \frac{\lambda_{j}}{2}\right) \sin \omega t\right),
\end{aligned}
$$

and its absolute magnitude can be derived to be [41]

$$
\begin{aligned}
|z(x)|=\frac{4 W}{m l} \sum_{j}^{\infty} \frac{\sin \lambda_{j} x}{\lambda_{j} \omega_{j}^{2} \sqrt{\left[\left(1-\frac{\omega^{2}}{\omega_{j}^{2}}\right)^{2}+\left(2 \zeta_{j} \frac{\omega}{\omega_{j}}\right)^{2}\right]}} . \\
\left(\left(\sin (b+a) \frac{\lambda_{j}}{2} \cdot \sin (b-a) \frac{\lambda_{j}}{2}\right) \sin \omega t\right) .
\end{aligned}
$$

To summarize, the physics-based process modeling of FFF extruder with regards to nozzle clogging was developed by analyzing the bar mount as Euler-Bernoulli 
boundary conditions of the beam were specified to be pinned at both ends, and the modal solution for the lateral displacement of the beam was obtained.

\subsection{Nozzle condition monitoring experiment preparation}

The experiment preparation consists of two main steps, namely, development of a $3 \mathrm{D}$ printer suitable for vibration monitoring tasks, and preparation for the nozzle condition monitoring experiment.

\subsubsection{Development of novel 3D printer for nozzle condition monitoring}

Proposed nozzle condition monitoring method using vibration sensor requires placing the accelerometer on the bar mount of an extruder. Currently, most platform along three axes allows installing any type of extruder, for instance, Direct or Bowden. The prototype of the 3D printer with Cartesian parallel printing platform and a fixed extruder built for the current study is presented in Figure 7. The development of the 3D printer was performed as follows. for easy installation/replacement of the Direct or Bowden types of extruders. This was performed by using aluminum profile together with universal brackets for clamping Direct extruder's stepper motor and Bowden extruder's stepper 
motor with the bar mount via bolts and nuts. Secondly, the printing platform were extruded individually via Direct and Bowden extruders while changing the extrusion temperature from $220^{\circ} \mathrm{C}$ to $170^{\circ} \mathrm{C}$ with the step size of $10^{\circ} \mathrm{C}$. 
Meanwhile, the filament extrusion process was recorded via JVC TK-C1480BE the nozzle end. The images of the filament extrusion process were extracted from the 25 frames per second video with the size of $1280 \times 720$ pixels and the resolution of 3 microns per pixel. After processing the recorded images using MATLAB software, 50 measurements were taken for each step of nozzle condition. effective nozzle diameter. The mean values of effective nozzle diameter for each temperature parameter were used to simulate the amplitude of the bar mount's vibration using the proposed theoretical model and then compared with the measured data gathered from the accelerometer.

Notably, the nozzle temperature reduction during extrusion was used only as a method of simulating the nozzle clogging, because decreasing the nozzle temperature solidifies the filament around the inner walls of the nozzle which leads to the reduction of filament diameter. Authors note that such drastic extrusion temperature decrease is not likely to happen during actual 3D printing, but was used for nozzle clog simulation purposes only. In actual 3D printing, a nozzle can start to clog because of different reasons such as the presence of dust inside a liquefier, absence of space for extrusion due to insufficient layer height or warping of printing part [45], and others.

\subsection{Simulation of the clogged nozzle}

Simulation of the clogged nozzle was obtained by taking the values of the filament diameters during temperature reduction, i.e., partially blocked nozzle. Furthermore, the simulation of the bar mount vibration was performed by calculating the natural frequency of the system and derivation of the Fourier amplitude spectrum of acceleration for the partially blocked nozzle. The process parameters used in the present study are listed in Table 1. The simulation of the nozzle clogging consists of the following steps.

- The nozzle clogging simulation was done by taking the nozzle diameter values from the temperature reduction experiment. This was performed 
by measuring filament diameters during extrusion from 220 to $170^{\circ} \mathrm{C}$ temperatures, by taking 50 measurements for each step of nozzle condition and estimating the mean values.

- The natural frequency of the system was calculated from equation (17) using parameters listed in Table 1.

- The Fourier amplitude spectrum of acceleration was obtained from equation (20) using parameters listed in Table 1 for the partially blocked nozzle diameter values.

After calculating the frequency response of the bar mount for Direct and Bowden extruder, the data were saved, and the resultant graphs were plotted, which are discussed in the Results section.

\subsection{Experimental procedure}

The experimental procedure includes the placement of the accelerometer to the extruder and the overall experimental setup that was used for nozzle condition monitoring using the vibration sensor [46]. The accelerometer installation on Direct and the Bowden extruder are shown in Figure 8 and Figure 9. The layout of the experimental setup is illustrated in Figure 10. The accelerometer was mounted on the bar mount from the bottom and connected to a charge amplifier. The accelerometer data capturing was not significantly affected by the heat from the liquefier heater block. This is because the maximum allowed operating temperature for the used accelerometer is $250^{\circ} \mathrm{C}$ and the bar mount temperature was constantly below $100^{\circ} \mathrm{C}$. After signal amplification, the data was processed using a signal analyzer and was sent to the personal computer. The experimental setup can be described as follows.

- Accelerometer: Bruel and Kjaer 4393 Piezoelectric charge accelerometer with mass of $2.4 \mathrm{~g}$, and sensitivity of $4.8 \mathrm{mV} / \mathrm{g}$. The accelerometer was placed on the bottom side of the bar mount. 
- Charge amplifier: Bruel and Kjaer Charge Amplifier type 2635 with output unit set as acceleration in $\mathrm{m} / \mathrm{s}^{2}$.

- Signal analyser: Hewlett Packard 35670a Dynamic Signal Analyser.

Nozzle condition monitoring experiment process parameters are listed in Table 1.

355 The vibration signals gathered from the accelerometer were analyzed in time and frequency domain.

\section{Results}

\subsection{Nozzle diameter during partial clogging due to temperature reduction}

The mean values of effective nozzle diameter at the range of extrusion temperatures for Direct and Bowden extruders are illustrated in Figure 11 and Figure 12 respectively. As can be seen, the decrease in the nozzle temperature decreased the actual nozzle diameter, which simulated a clogged nozzle condition during filament extrusion. For three filaments and two extruder types used in the current experiment, the nozzle diameter reduction showed nonlinear behavior to the nozzle temperature reduction. There are two main observations. First, the nozzle clogging simulation results for both Direct and Bowden extruders showed that the ABS filament was less sensitive to the changes in nozzle temperature. Also, ABS filament was subjected to a minimum decrease in effective nozzle diameter in comparison to the PLA and SemiFlex filaments. On the other hand, the SemiFlex filament was more sensitive to the nozzle temperature reduction and was subjected to a maximum nozzle diameter decrease among the three analyzed filaments. Second, the mean values of effective nozzle diameter decreased more for the Bowden extruder during simulated nozzle clogging. Authors note that in the current study the Direct extruder had a $0.4 \mathrm{~mm}$ nozzle diameter, while the Bowden extruder had a $0.35 \mathrm{~mm}$ nozzle diameter, and that is why the comparison between these two extruders was performed by estimating the percentage decrease from their nominal nozzle diameter values. Thus, the percentage decrease of the mean values of effective nozzle diameter during simulated nozzle clogging were: 
- Direct extruder: ABS: 12\%; b) PLA: 13\%; SemiFlex: 14\%;

380

Considering this, the Bowden extruder tended to clog more than the Direct extruder in the present work, especially when using a SemiFlex filament. To sum up, the present experiment showed that ABS filament tended to clog less than PLA and SemiFlex filaments, and the Direct extruder was less prone to nozzle clogging in comparison to the Bowden extruder.

\subsection{Natural frequency and frequency spectrum}

The theoretical and experimental results of the natural frequency and acceleration amplitudes for extruding PLA filament via Direct and Bowden extruders are shown in Table 2 and Table 3 respectively. The Fourier amplitude spectra of acceleration near to the natural frequency were derived theoretically and compared with the experimental data for PLA filament extrusion via Direct and Bowden extruders for each nozzle diameter during clogging, and the differences were found to be in the range of $5 \%$. Therefore, the proposed theoretical model is relatively adequate for calculating the natural frequency and the acceleration amplitude of the bar mount vibration during filament extrusion.

The theoretical and experimental results of the Fourier acceleration amplitude spectra for extruding PLA filament via Direct and Bowden extruders during clogging simulation are presented in Figure 13 and Figure 14 respectively. As can be seen, the Fourier amplitude spectrum of acceleration increased nonlinearly with the reduction of the nozzle diameter. For example, for the Direct extruder's nozzle clogging from $0.4 \mathrm{~mm}$ to $0.34 \mathrm{~mm}$ the amplitude of acceleration near the natural frequency value increased from 0.615 to $1.068 \mathrm{~m} / \mathrm{s}^{2}$ during theoretical calculation, and from 0.647 to $1.112 \mathrm{~m} / \mathrm{s}^{2}$ during experiments, or $73.54 \%$ and $71.75 \%$ rise respectively. Thus, the theoretical calculations on the relative increase in the vibrations of the bar mount during nozzle clogging are relatively accurate. 
The experimental peaks of the bar mount's acceleration amplitudes at about the natural frequency value using PLA, ABS and SemiFlex filaments on Direct and Bowden extruders are shown in Figure 15 and Figure 16 respectively. As can be seen, the reduction of the effective nozzle diameter increased the bar mount's transverse vibrations. This behavior was calculated theoretically using the proposed beam model and the results are shown in Figure 13, Figure 14 and Table 2, Table 3. Besides, Figure 15 and Figure 16 show that extrusion of the PLA filament forces the bar mount to vibrate more for both extruders in comparison with ABS and SemiFlex filaments. This can be explained by the fact that the PLA filament has the highest Young's modulus and requires more feeding force to push through the liquefier. To summarize, the results illustrate that the proposed theoretical model adequately represents the nozzle clogging condition in FFF. The proposed technique showed a high correlation of the theory with the experiments in terms of several parameters, such as natural frequency, acceleration amplitude increase with the nozzle diameter decrease, and the non-linear trend of acceleration amplitude change for three types of filaments materials and two types of extruders.

\section{Discussions}

The findings from theoretical and experimental results show that the proposed technique can predict the nozzle condition relatively accurately for main types of FFF extruders and filaments. The natural frequency values calculated from the theoretical model were very near to the values obtained from the experiments. Further, the amplitudes of acceleration derived from the theoretical relationships were comparatively close to the experimental data gathered from the accelerometer. In addition, the theoretical model proposed that the bar mount of the FFF extruder will vibrate more when the nozzle becomes clogged, which was verified from the set of experiments conducted using Direct and Bowden extruder types. Moreover, the physics-based model predicted the non-linear trend of the 
behavior during extruding PLA, ABS, and SemiFlex filaments via Direct and Bowden extruders. Therefore, the proposed technique is relatively adequate for predicting nozzle clogging condition in the FFF 3D printers.

The results of the current study can be significant for monitoring nozzle clogging in FFF 3D printing as the proposed model is based on the physics-based relationships between process parameters and nozzle condition. The findings of the present research are relatively close to the study reported earlier by Bukkapatnam and Clark [36] on dynamic modeling and monitoring of process errors in layered AM process. Their work focused more on capturing severe disturbances of the 3D printer structure, whereas current study concentrates more on the vibration of the extruder and tracks the nozzle clogging condition. Another significant aspect of the current study is relatively accurate sensor placement. The accelerometer was mounted on the bar mount of the FFF extruder, which is in contrast to the earlier studies reported on condition monitoring in layered AM ([33], [34], [35], [36]). Also, the proposed vibration measurement technique is close to the method presented by Go et al. [47], where they fastened a plate with an attached strain gauge to the bar mount to measure the maximum force exerted by the feed mechanism on the filament.

There are several limitations of the present study. First, theoretical calculation results of the applied forces were slightly different from the experimental results. This is because the theoretical model consists of a process parameter as the radius of the filament, which was assumed ideally constant. However, the actual radius of the filament tends to vary during extrusion due to the filament's manufacturing limitations and tolerances, for example, tolerance of filaments used in present study was $+/-0.05 \mathrm{~mm}$. To overcome this limitation, the actual radius of the filament can be measured at the location before it enters the heated liquefier. The actual filament radius measurement can be achieved using computer vision system, similar as in work presented by Greeff and Schilling [29], where they measured the filament width using a low-cost USB microscope video camera and image processing. Then, the actual radius of filament values $R_{1}$ should be placed in equations (3) and (4) and subsequently used for estimating 
the natural frequency amplitude of acceleration of the bar mount. Second, the theoretical calculation results of the acceleration amplitudes were tolerably lower than the experimental results. This is because the mass of the wires connecting to the heater block, temperature sensors, and accelerometers was not included in the theoretical model.

Authors are currently extending the proposed method to desktop 3D printer extruders that move during fabrication using low-cost sensors, and preliminary results are encouraging. In particular, a $3 \mathrm{D}$ printer used for the preliminary study was MakerBot Replicator 1 with Direct extruder based on Cartesian gantry mechanism. Next, the vibrations of the bar mount were captured using low-cost ADXL001 MEMS accelerometer with a sensitivity of $6.7 \mathrm{mV} / \mathrm{g}$. This MEMS accelerometer was fixed to EVAL-ADXL001 PCB board and connected to EVAL-CN0303-SDPZ MEMS-based vibration analyzer. The vibration analyzer converted the output voltage of MEMS accelerometer into a digital signal using AD7476 analog-to-digital converter. The total cost of a MEMS accelerometer, a PCB board, and a MEMS-based vibration analyzer was below US $\$ 200$, which is much less than the piezoelectric sensor and the dynamic signal analyzer used in the present study. The accelerometer installation on the 3D printer is presented in Figure 17. Preliminary experiments of nozzle condition monitoring were performed by measuring bar mount vibrations during 3D printing different objects using PLA, ABS, SemiFlex filaments for a long period of time until the nozzle was completely clogged. The vibration signals were analyzed in a frequency domain, the natural amplitudes of bar mount acceleration were captured, and the disturbances caused by the extruder movement dynamics and inertia were not allowed to pass using a digital notch filter. As can be seen from Figure 18, completely clogged nozzle increased the amplitudes of acceleration drastically, which showed a similar trend with our proposed theoretical and simulation results.

Future work may address the usage of the proposed technique for real-time nozzle condition monitoring and control in $3 \mathrm{D}$ printing using various process parameters and filament materials. 


\section{Conclusion}

Nozzle clogging is one of the most significant process errors in 3D printers,

500 surface roughness, and mechanical properties. This study proposes a nozzle condition monitoring technique in FFF 3D printing using a vibration sensor. The proposed technique is based on a physical model of the FFF process with respect to nozzle condition and was used to monitor nozzle clogging during extrusion

In conclusion, the findings of the current work can be one step towards developing nozzle condition monitoring in $3 \mathrm{D}$ printing as the study was based on the fundamental relationships between FFF process parameters and the nozzle clogging.

- Nozzle condition can be monitored using an accelerometer by measuring the vibrations of a bar mount that supports a liquefier block in Direct or Bowden FFF extruder.

- To monitor the nozzle condition, the boundary conditions of a bar mount required to be set accordingly. The bar mount of Direct and the Bowden FFF extruder was modeled as a pinned-pinned beam excited by a uniform loading distributed over a partial length.

- Theoretical and experimental results obtained in the current study for PLA, ABS, and SemiFlex filament materials extruded via Direct and Bowden type of FFF extruders showed that the acceleration amplitudes of the bar mount increased non-linearly during nozzle clogging. 
References

[1] F42-Committee, Terminology for additive manufacturing technologies, ASTM International.

[2] S. S. Crump, Apparatus and method for creating three-dimensional objects, US Patent 5,121,329 (Jun. 9 1992).

[3] A. Bowyer, 3D printing and humanity's first imperfect replicator, 3D printing and additive manufacturing 1 (1) (2014) 4-5.

[4] R. Jones, P. Haufe, E. Sells, P. Iravani, V. Olliver, C. Palmer, A. Bowyer, Reprap-the replicating rapid prototyper, Robotica 29 (1) (2011) 177-191.

[5] B. T. Wittbrodt, A. Glover, J. Laureto, G. Anzalone, D. Oppliger, J. Irwin, J. M. Pearce, Life-cycle economic analysis of distributed manufacturing with open-source 3-D printers, Mechatronics 23 (6) (2013) 713-726.

[6] C. Schelly, G. Anzalone, B. Wijnen, J. M. Pearce, Open-source 3-D printing technologies for education: Bringing additive manufacturing to the classroom, Journal of Visual Languages \& Computing 28 (2015) 226-237.

[7] K. Telegenov, Y. Tlegenov, A. Shintemirov, A low-cost open-source 3-dprinted three-finger gripper platform for research and educational purposes, IEEE access 3 (2015) 638-647.

[8] I. Campbell, D. Bourell, I. Gibson, Additive manufacturing: rapid prototyping comes of age, Rapid prototyping journal 18 (4) (2012) 255-258.

[9] I. Gibson, D. Rosen, B. Stucker, Additive Manufacturing Technologies: 3D Printing, Rapid Prototyping, and Direct Digital Manufacturing, Springer, 2014.

[10] S. Guo, Y. He, L. Shi, S. Pan, R. Xiao, K. Tang, P. Guo, Modeling and experimental evaluation of an improved amphibious robot with compact structure, Robotics and Computer-Integrated Manufacturing 51 (2018) $37-52$. 
[11] Y. Tlegenov, K. Telegenov, A. Shintemirov, An open-source 3D printed underactuated robotic gripper, in: Mechatronic and Embedded Systems and Applications (MESA), 2014 IEEE/ASME 10th International Conference on, IEEE, 2014, pp. 1-6.

[12] J. M. Pearce, Building research equipment with free, open-source hardware, Science 337 (6100) (2012) 1303-1304.

[13] J. M. Pearce, Open-source lab: how to build your own hardware and reduce research costs, Elsevier, 2014.

[14] I. Zein, D. W. Hutmacher, K. C. Tan, S. H. Teoh, Fused deposition modeling of novel scaffold architectures for tissue engineering applications, Biomaterials 23 (4) (2002) 1169-1185.

[15] S. J. Kalita, S. Bose, H. L. Hosick, A. Bandyopadhyay, Development of controlled porosity polymer-ceramic composite scaffolds via fused deposition modeling, Materials Science and Engineering: C 23 (5) (2003) 611-620.

[16] Y. Wang, R. Blache, P. Zheng, X. Xu, A knowledge management system to support design for additive manufacturing using bayesian networks, Journal of Mechanical Design 140 (5).

[17] A. Boschetto, L. Bottini, F. Veniali, Finishing of fused deposition modeling parts by CNC machining, Robotics and Computer-Integrated Manufacturing $41(2016) 92-101$.

[18] S.-H. Ahn, M. Montero, D. Odell, S. Roundy, P. K. Wright, Anisotropic material properties of fused deposition modeling abs, Rapid Prototyping Journal 8 (4) (2002) 248-257.

575

[19] B. Tymrak, M. Kreiger, J. M. Pearce, Mechanical properties of components fabricated with open-source 3-d printers under realistic environmental conditions, Materials \& Design 58 (2014) 242-246. 
[20] S. Costa, F. Duarte, J. Covas, Estimation of filament temperature and adhesion development in fused deposition techniques, Journal of Materials Processing Technology 245 (2017) 167-179.

[21] M. Kaveh, M. Badrossamay, E. Foroozmehr, A. H. Etefagh, Optimization of the printing parameters affecting dimensional accuracy and internal cavity for hips material used in fused deposition modeling processes, Journal of Materials processing technology 226 (2015) 280-286.

[22] C. Yang, X. Tian, D. Li, Y. Cao, F. Zhao, C. Shi, Influence of thermal processing conditions in $3 \mathrm{~d}$ printing on the crystallinity and mechanical properties of peek material, Journal of Materials Processing Technology 248 (2017) $1-7$.

[23] C. Kim, D. Espalin, A. Cuaron, M. A. Perez, E. MacDonald, R. B. Wicker, A study to detect a material deposition status in fused deposition modeling technology, in: 2015 IEEE International Conference on Advanced Intelligent Mechatronics (AIM), IEEE, 2015, pp. 779-783.

[24] J. Straub, Initial work on the characterization of additive manufacturing (3d printing) using software image analysis, Machines 3 (2) (2015) 55-71.

[25] J. Straub, Characterization of internal geometry/covered surface defects with a visible light sensing system, in: Image Sensing Technologies: Materials, Devices, Systems, and Applications III, Vol. 9854, International Society for Optics and Photonics, 2016, p. 98540W.

[26] A. Ceruti, A. Liverani, T. Bombardi, Augmented vision and interactive monitoring in 3d printing process, International Journal on Interactive Design and Manufacturing (IJIDeM) 11 (2) (2017) 385-395.

[27] S. Nuchitprasitchai, M. Roggemann, J. M. Pearce, Factors effecting realtime optical monitoring of fused filament 3d printing, Progress in Additive Manufacturing 2 (3) (2017) 133-149. 
[28] S. Nuchitprasitchai, M. C. Roggemann, J. M. Pearce, Three hundred and sixty degree real-time monitoring of 3-d printing using computer analysis of two camera views, Journal of Manufacturing and Materials Processing 1 (1) (2017) 2 .

[29] G. P. Greeff, M. Schilling, Closed loop control of slippage during filament transport in molten material extrusion, Additive Manufacturing 14 (2017) $31-38$.

[30] O. Holzmond, X. Li, In situ real time defect detection of 3D printed parts, Additive Manufacturing 17 (2017) 135 - 142.

[31] T. Fang, I. Bakhadyrov, M. A. Jafari, G. Alpan, Online detection of defects

[33] P. K. Rao, J. P. Liu, D. Roberson, Z. J. Kong, C. Williams, Online real-time quality monitoring in additive manufacturing processes using heterogeneous sensors, Journal of Manufacturing Science and Engineering 137 (6) (2015) 061007.

[34] H. Wu, Y. Wang, Z. Yu, In situ monitoring of FDM machine condition via acoustic emission, The International Journal of Advanced Manufacturing Technology 84 (5-8) (2016) 1483-1495.

[35] F. Baumann, M. Schön, J. Eichhoff, D. Roller, Concept development of a sensor array for 3D printer, Procedia CIRP 51 (2016) 24-31.

[36] S. Bukkapatnam, B. Clark, Dynamic modeling and monitoring of contour crafting - an extrusion-based layered manufacturing process, Journal of Manufacturing Science and Engineering 129 (1) (2007) 135-142. 
[37] B. N. Turner, R. Strong, S. A. Gold, A review of melt extrusion additive manufacturing processes: I. Process design and modeling, Rapid Prototyping Journal 20 (3) (2014) 192-204.

${ }_{635}$ [38] N. Venkataraman, S. Rangarajan, M. Matthewson, B. Harper, A. Safari, S. Danforth, G. Wu, N. Langrana, S. Guceri, A. Yardimci, Feedstock material property-process relationships in fused deposition of ceramics (FDC), Rapid Prototyping Journal 6 (4) (2000) 244-253.

[39] W. Michaeli, Extrusion dies for plastics and rubber, Carl Hanser Verlag GmbH \& Co. KG, 2003.

[40] A. Bellini, S. Guceri, M. Bertoldi, Liquefier dynamics in fused deposition, Journal of Manufacturing Science and Engineering 126 (2) (2004) 237-246.

[41] R. Barnoski, Response of elastic structures to deterministic and random excitation., Tech. rep., DTIC Document (1965).

${ }_{645}^{6}$ [42] Y. Tlegenov, Y. S. Wong, G. S. Hong, A dynamic model for nozzle clog monitoring in fused deposition modelling, Rapid Prototyping Journal 23 (2) (2017) 391-400.

[43] C. Gosselin, X. Kong, S. Foucault, I. Bonev, A fully-decoupled 3-dof translational parallel mechanism, in: Parallel Kinematic Machines in Research and Practice, 4th Chemnitz Parallel Kinematics Seminar, 2004, pp. 595-610.

[44] B. Danaei, N. Karbasizadeh, M. T. Masouleh, A general approach on collision-free workspace determination via triangle-to-triangle intersection test, Robotics and Computer-Integrated Manufacturing 44 (2017) 230 - 241.

[45] A. Armillotta, M. Bellotti, M. Cavallaro, Warpage of FDM parts: Experimental tests and analytic model, Robotics and Computer-Integrated Manufacturing 50 (2018) 140-152.

[46] J. Ratava, M. Lohtander, J. Varis, Tool condition monitoring in interrupted cutting with acceleration sensors, Robotics and Computer-Integrated Manufacturing 47 (2017) 70 - 75, sI: FAIM 2015. 
[47] J. Go, S. N. Schiffres, A. G. Stevens, A. J. Hart, Rate limits of additive manufacturing by fused filament fabrication and guidelines for high-throughput system design, Additive Manufacturing 16 (2017) 1 - 11. 


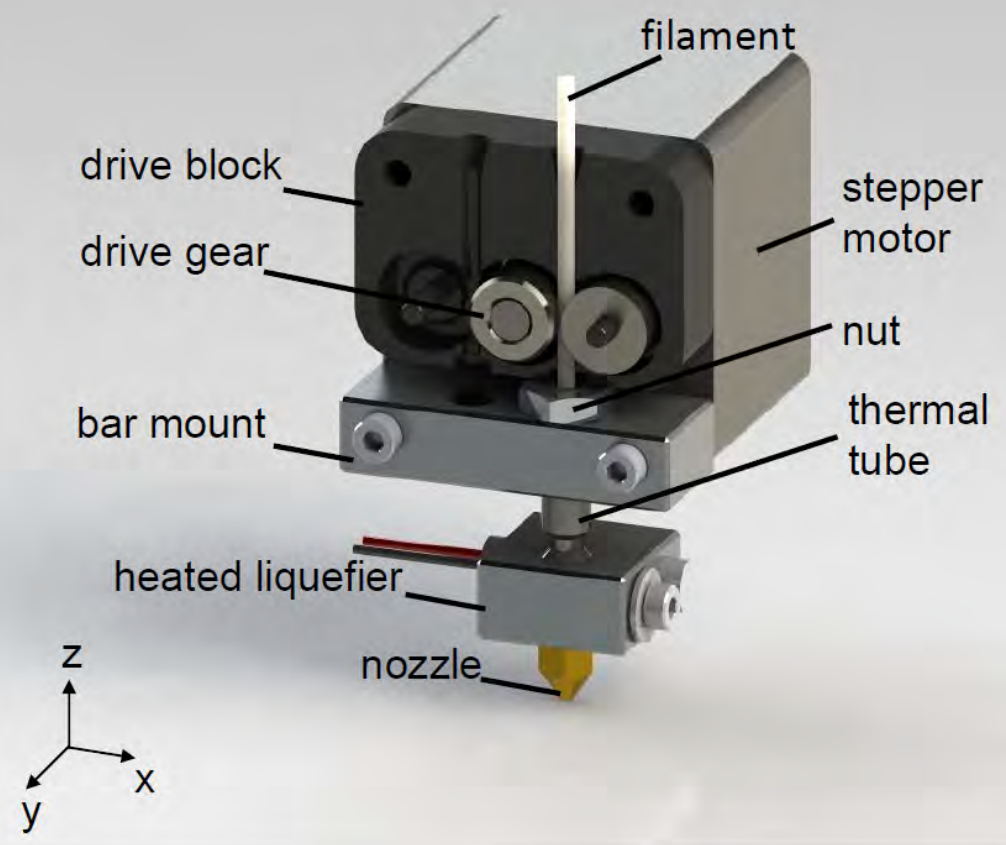

Figure 1: 3D layout of Direct FFF extruder. 


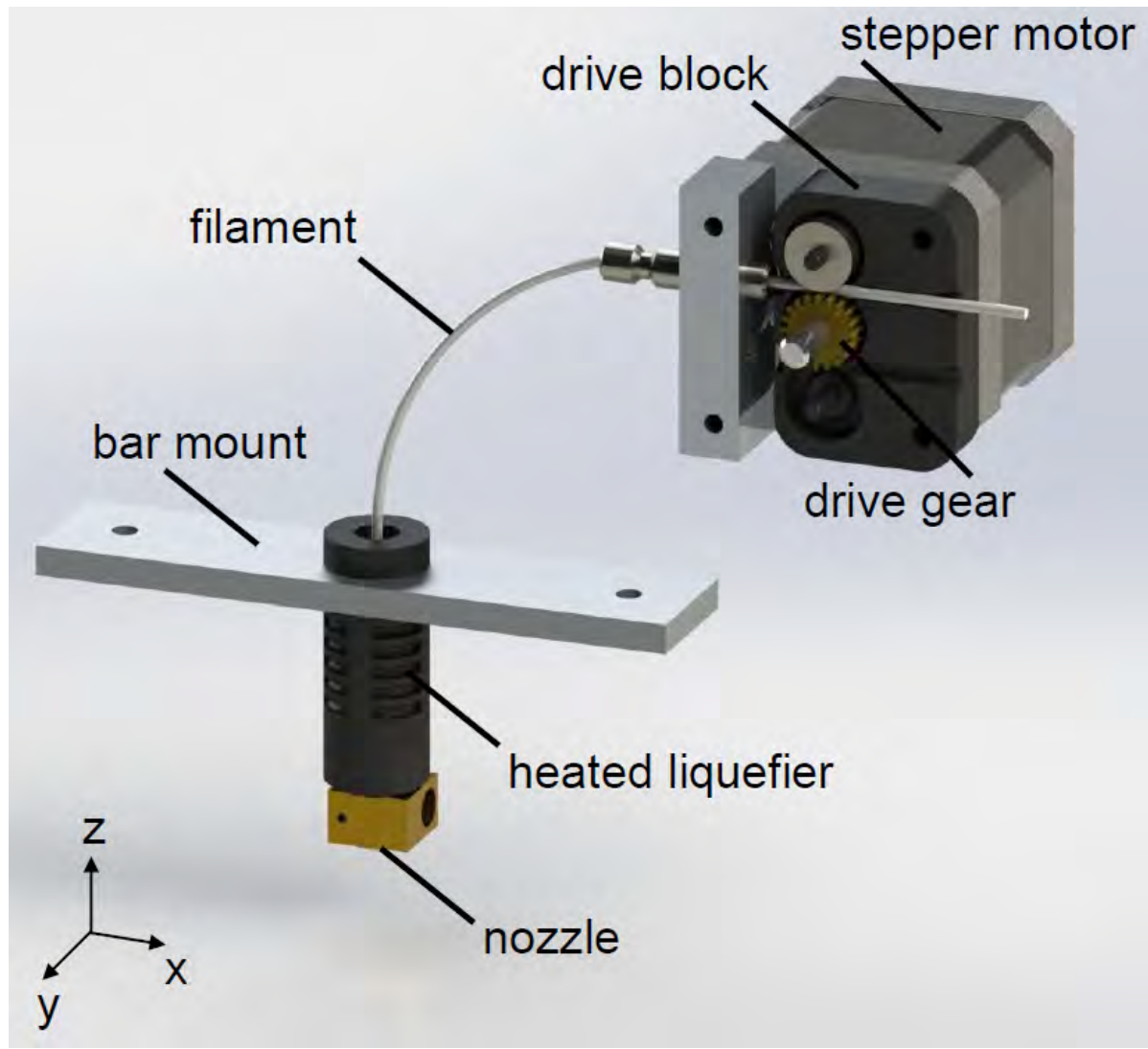

Figure 2: 3D layout of Bowden FFF extruder. 


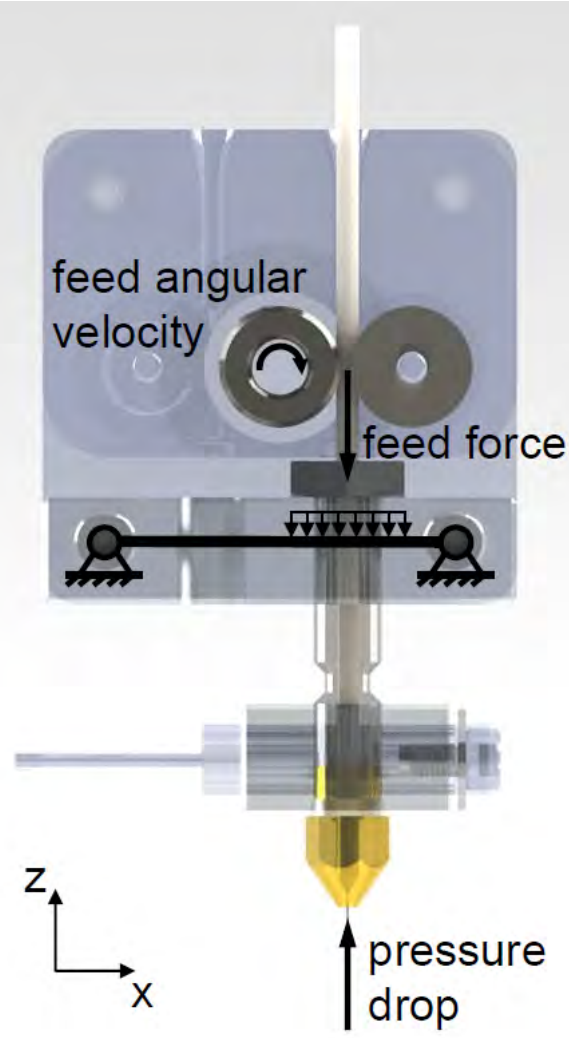

Figure 3: 2D layout of Direct FFF extruder with the beam model. 


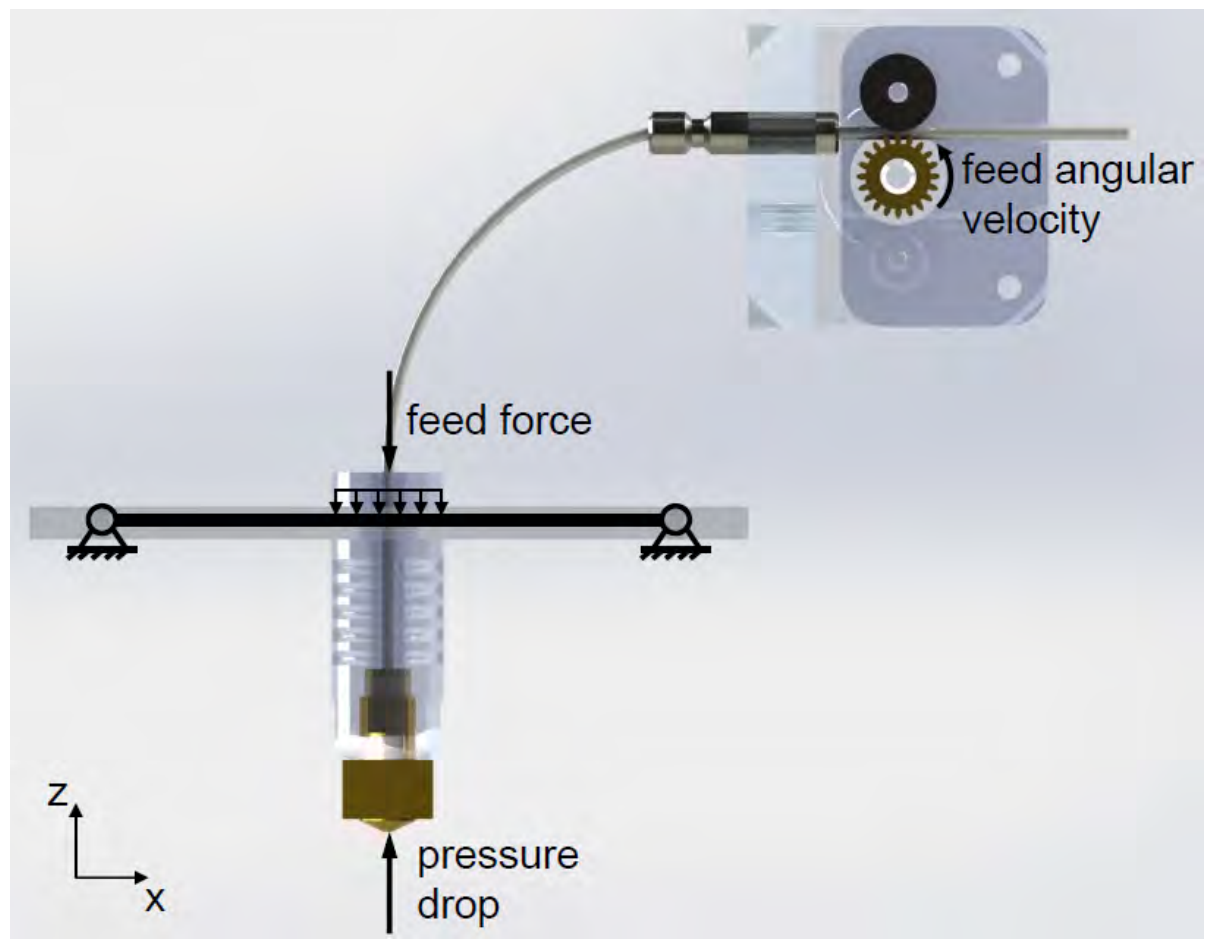

Figure 4: 2D layout of Bowden FFF extruder with the beam model. 


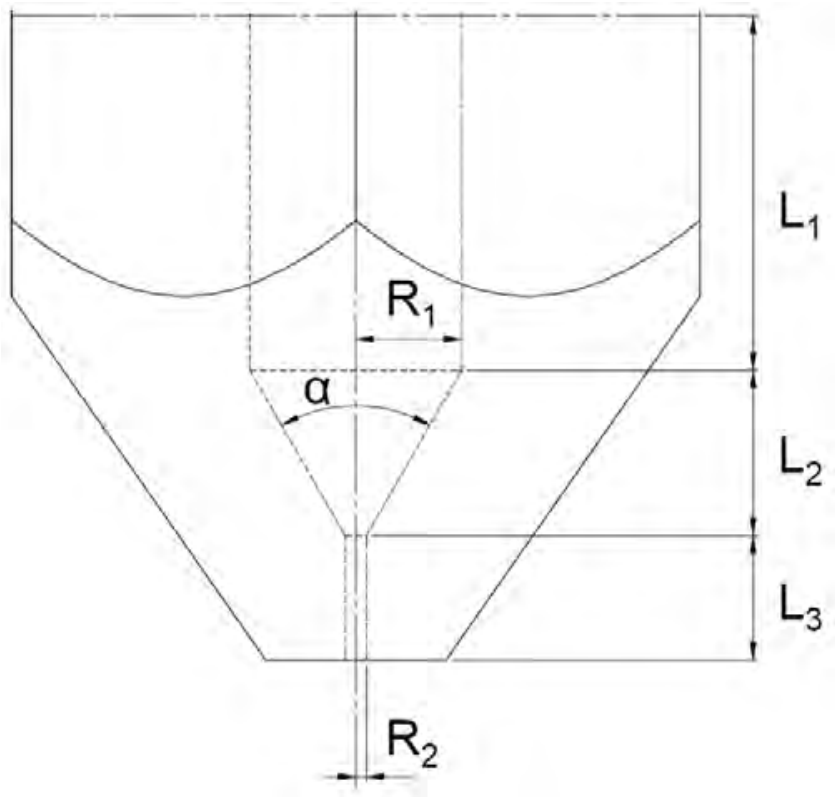

Figure 5: Nozzle layout with illustrated three zones. 


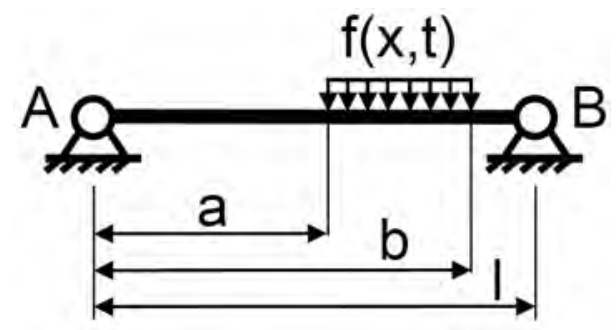

Figure 6: Pinned-pinned beam subjected to a partially distributed uniform loading. 


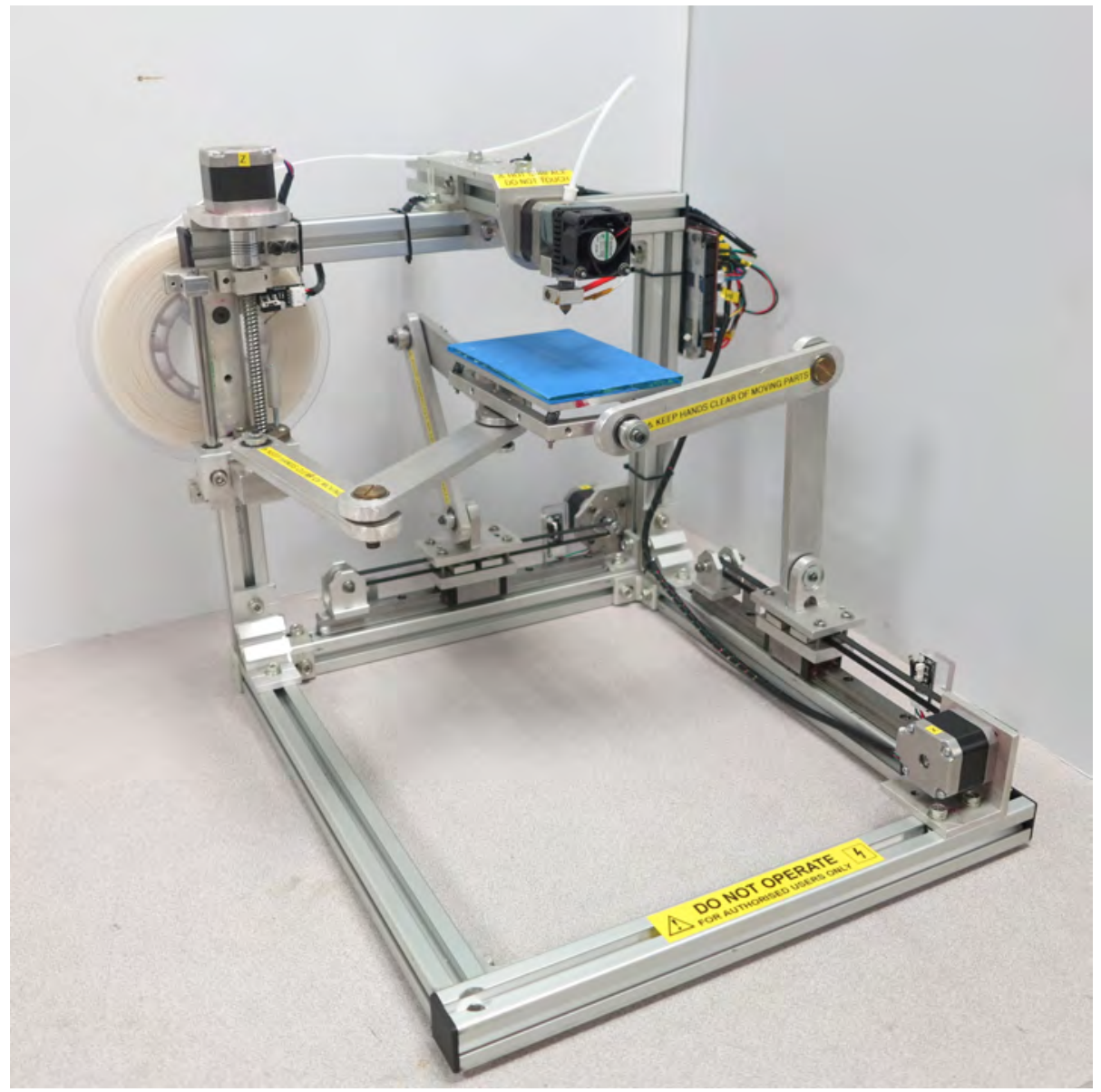

Figure 7: Novel 3D printer prototype with a moving printing platform and a fixed extruder. 


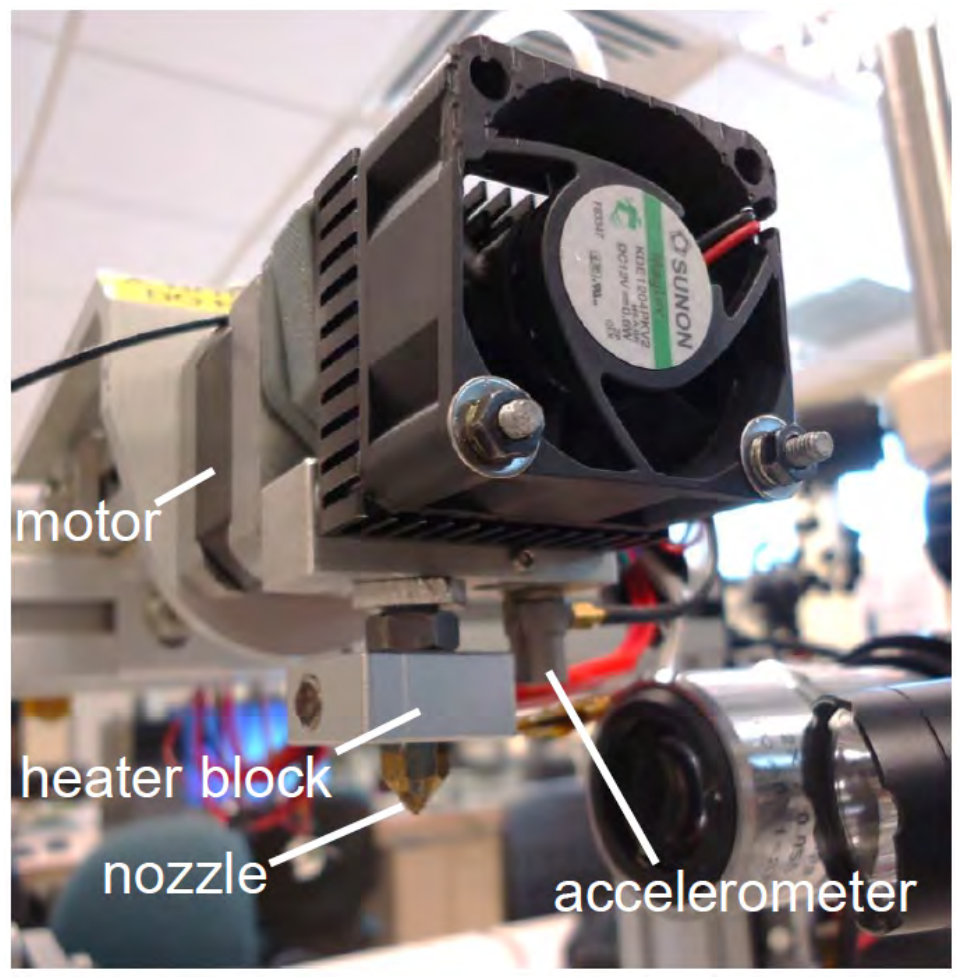

Figure 8: Accelerometer attachment to the bar mount of Direct extruder. 


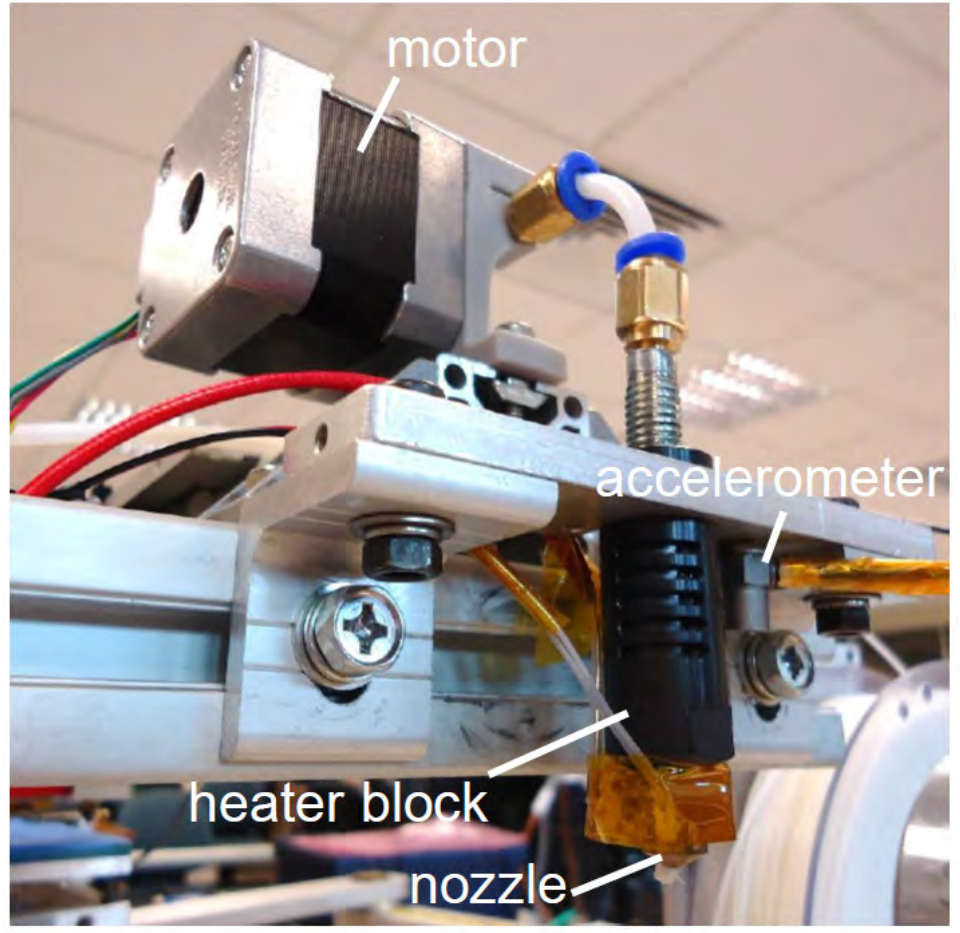

Figure 9: Accelerometer attachment to the bar mount of Bowden extruder. 


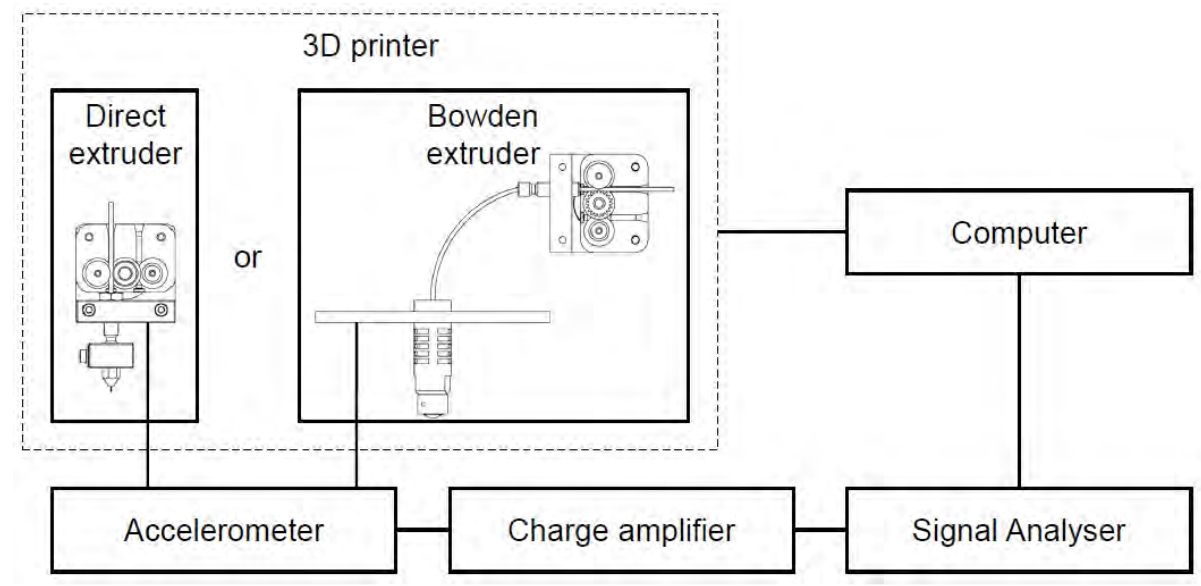

Figure 10: Experimental setup layout. 


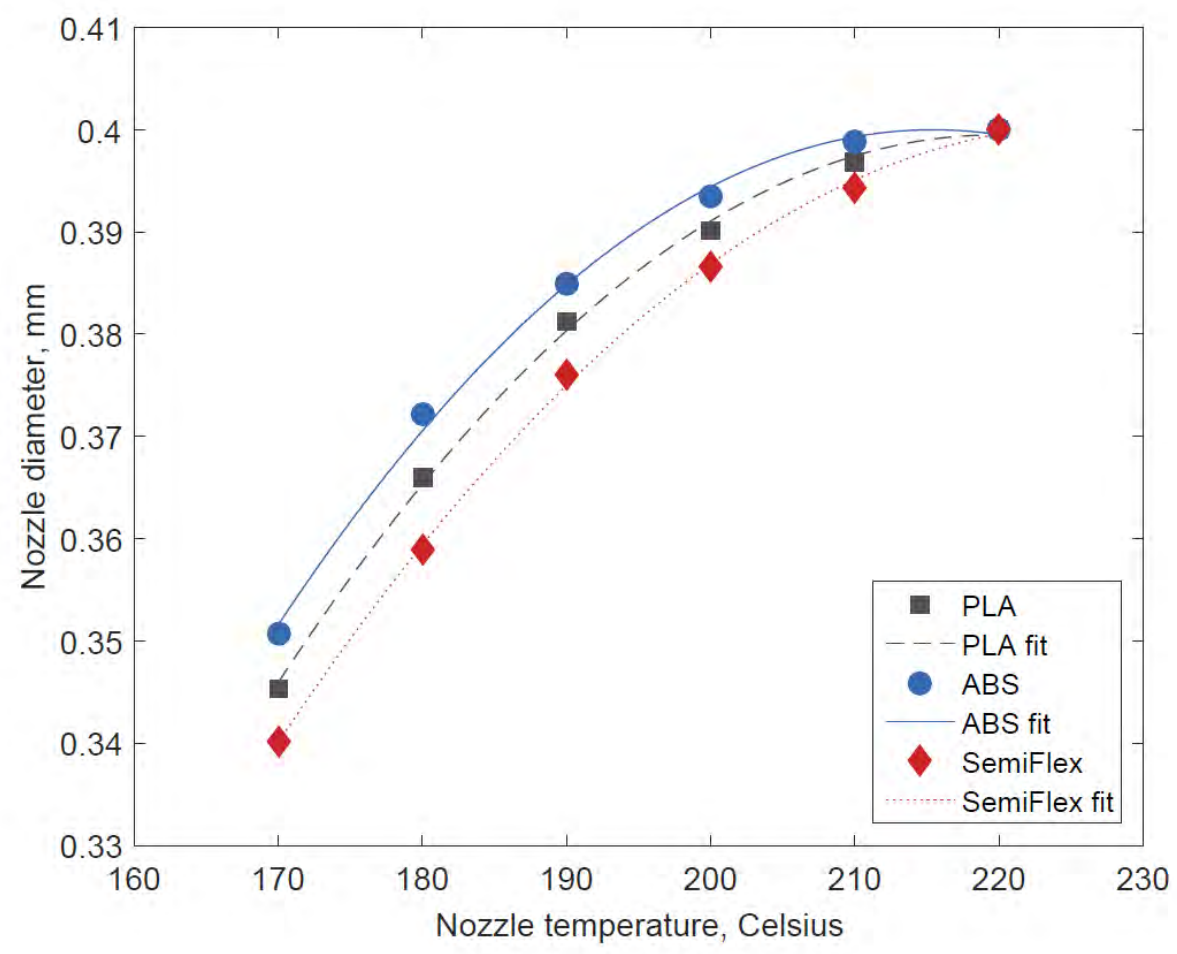

Figure 11: Mean values of effective nozzle diameter vs nozzle temperature in Direct extruder. 


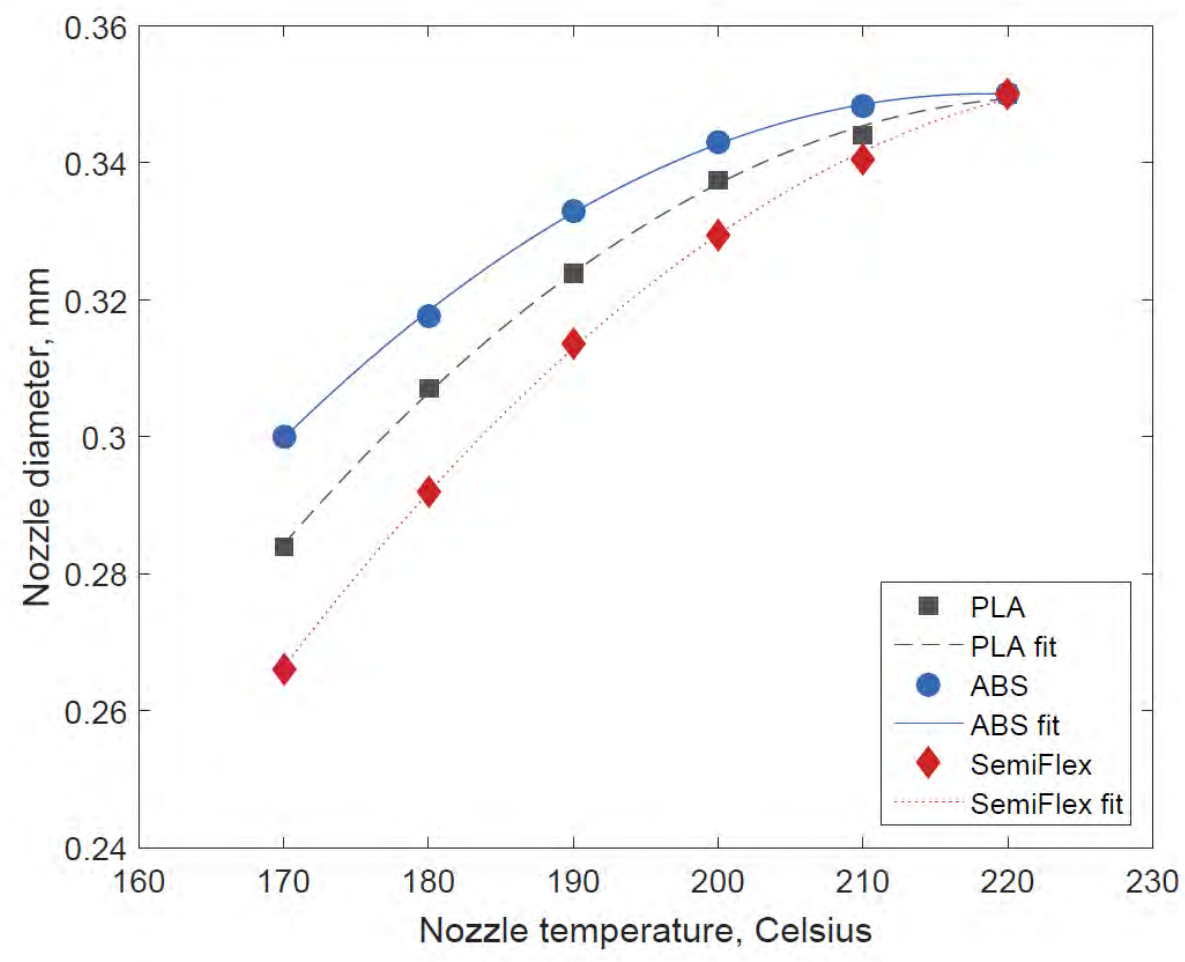

Figure 12: Mean values of effective nozzle diameter vs nozzle temperature in Bowden extruder. 


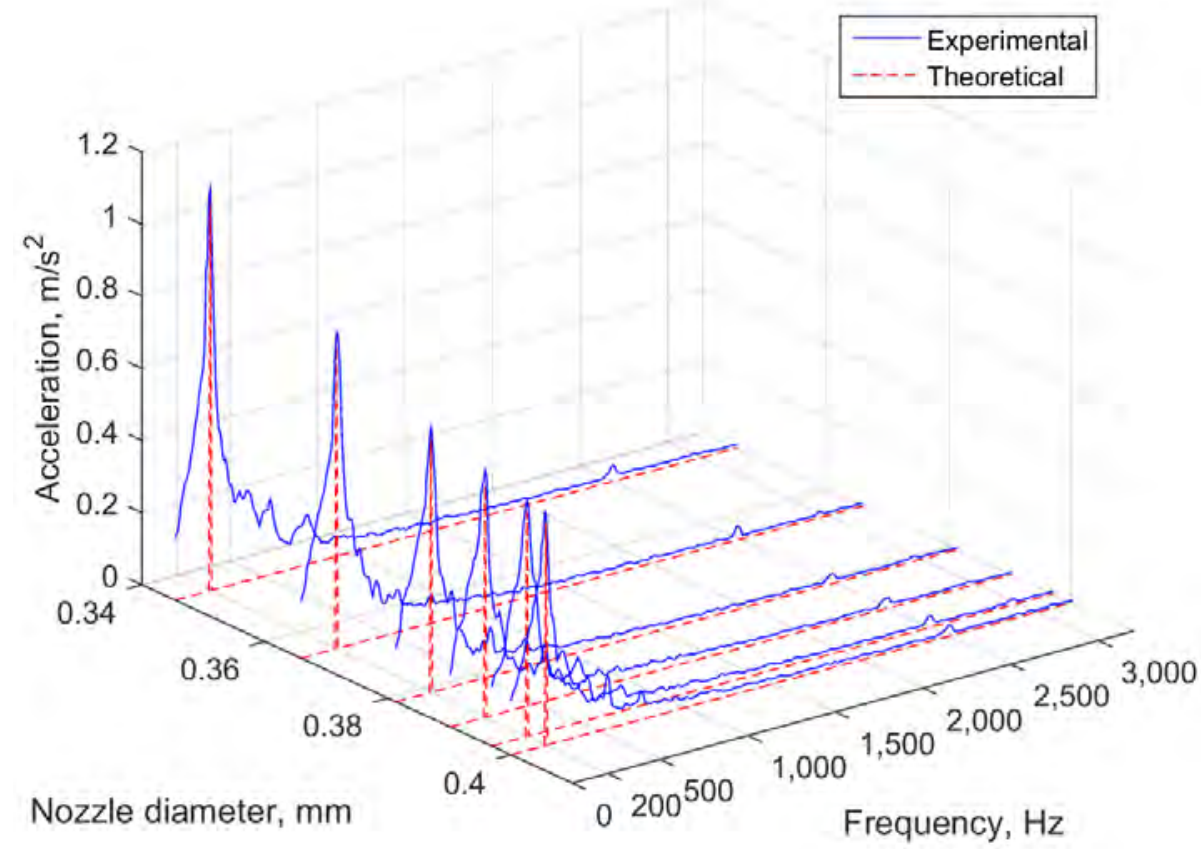

Figure 13: Theoretical and experimental acceleration amplitudes during nozzle clogging in Direct extruder, PLA filament. 


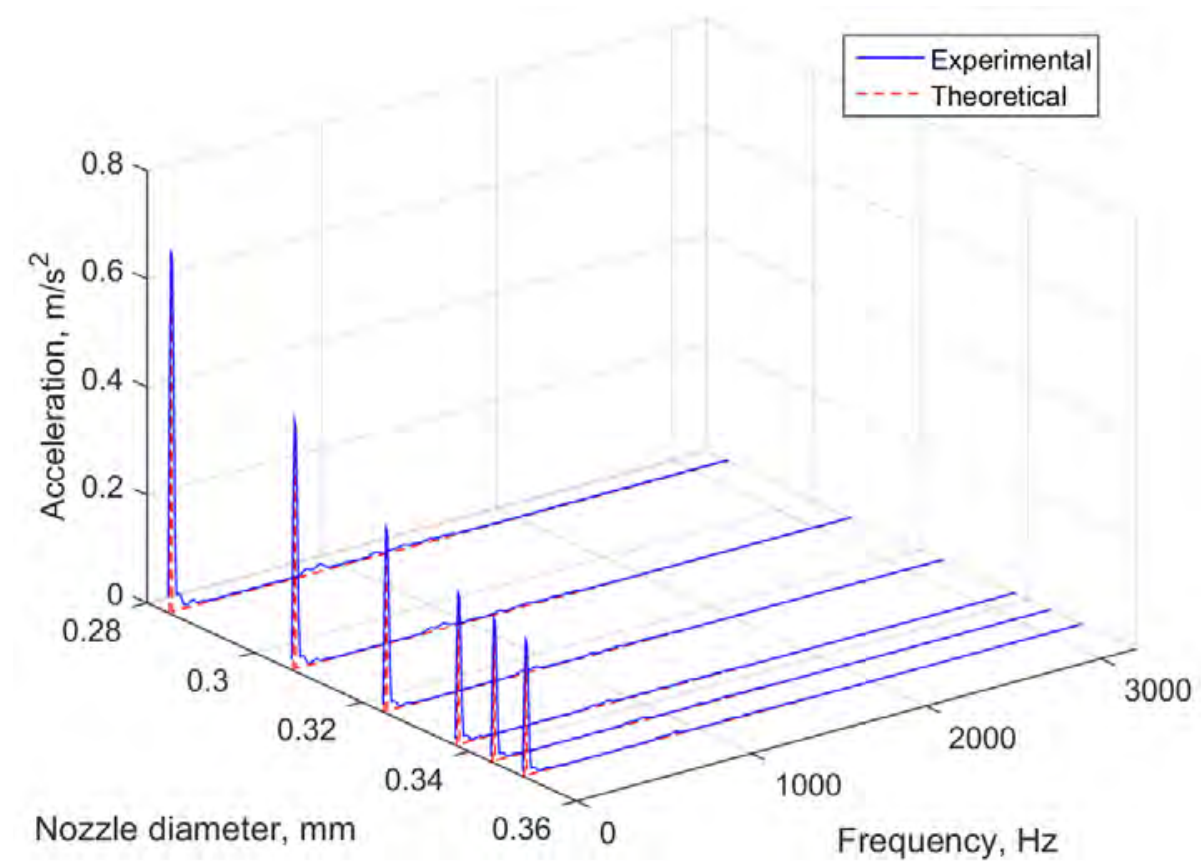

Figure 14: Theoretical and experimental acceleration amplitudes during nozzle clogging in Bowden extruder, PLA filament. 


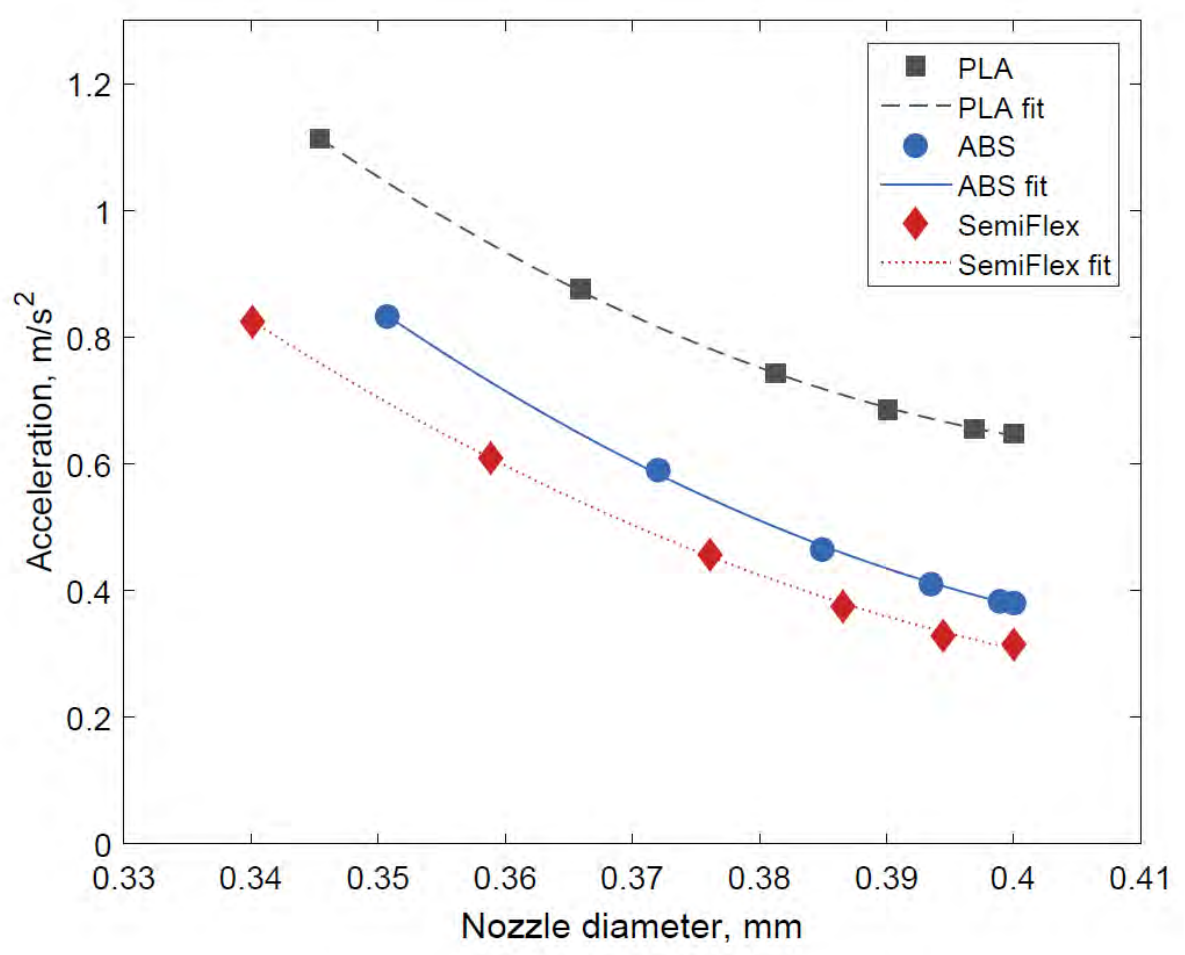

Figure 15: Experimental acceleration amplitudes during nozzle clogging in Direct extruder. 


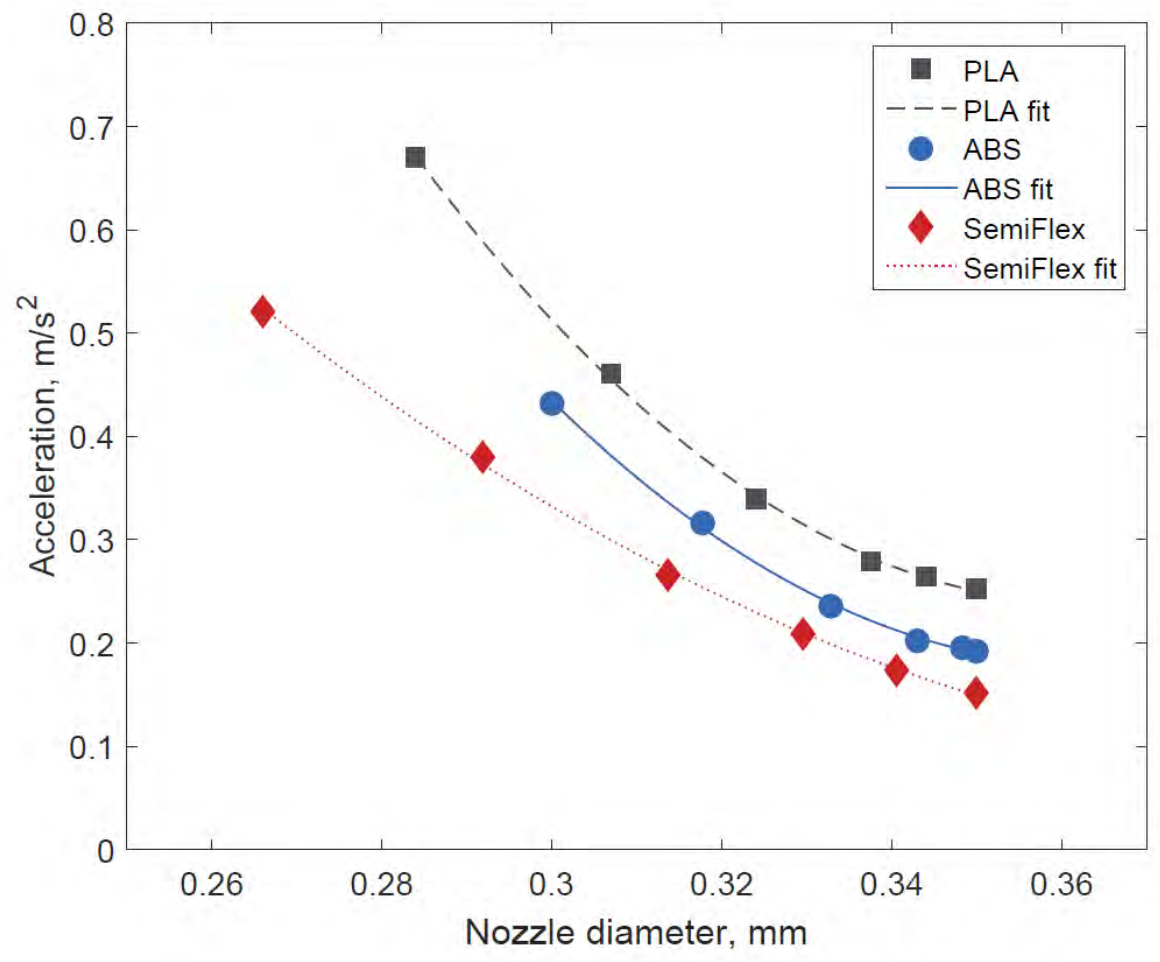

Figure 16: Experimental acceleration amplitudes during nozzle clogging in Bowden extruder. 


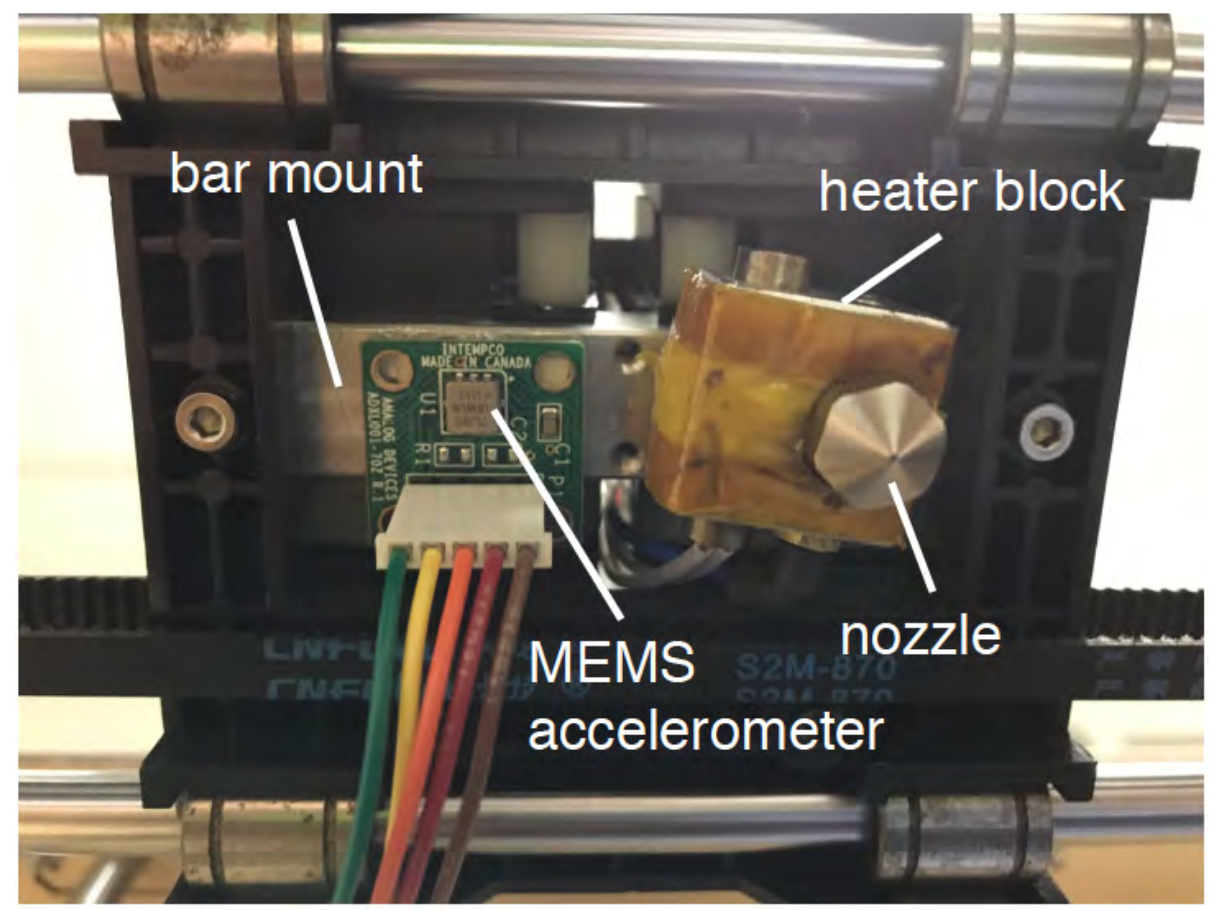

Figure 17: MEMS accelerometer attachment to the bar mount of Direct extruder of MakerBot Replicator $13 \mathrm{D}$ printer. 


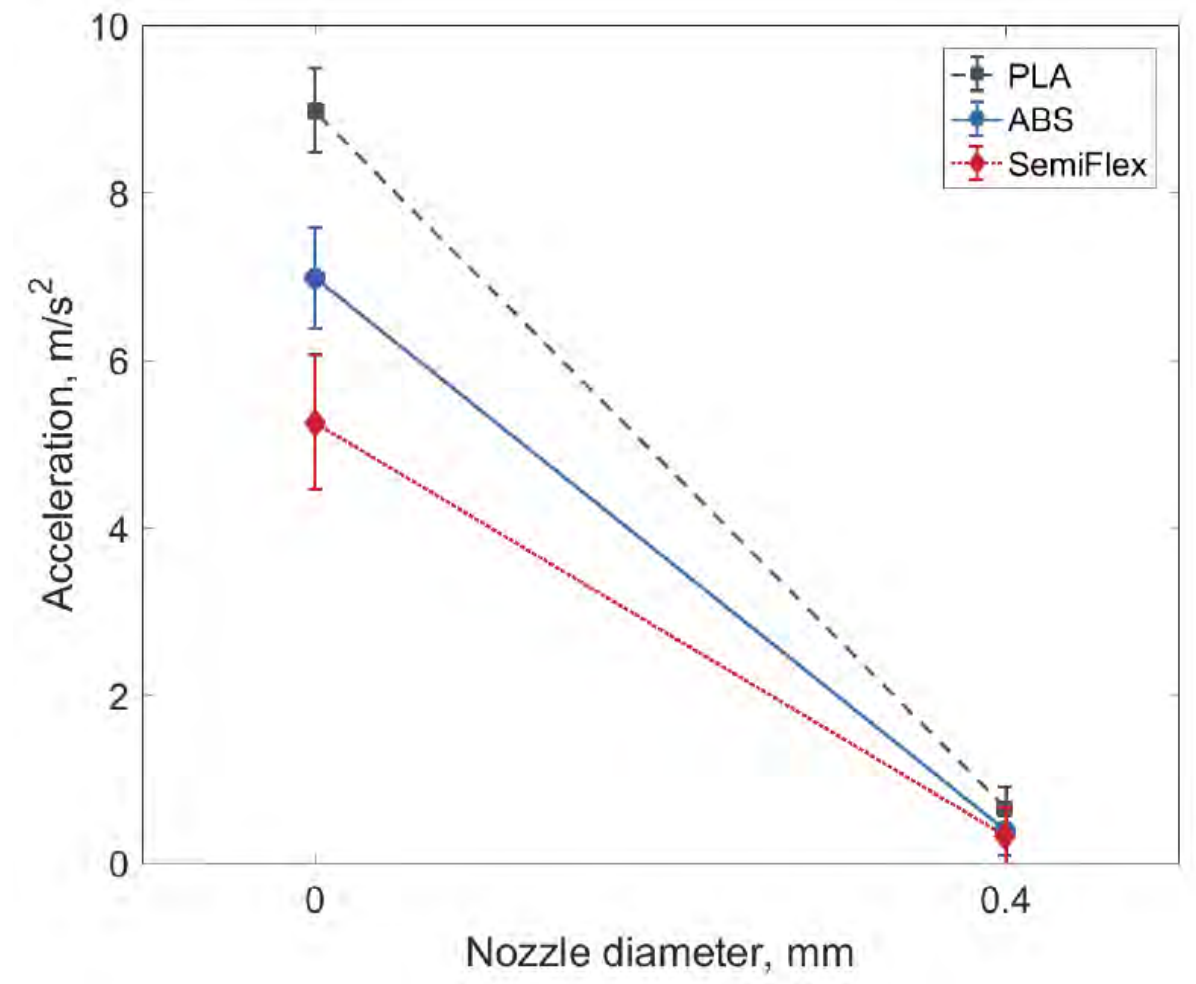

Figure 18: Experimental acceleration amplitudes during complete nozzle clogging in Direct extruder of MakerBot Replicator $13 \mathrm{D}$ printer. 
Table 1: Process parameters

\begin{tabular}{lc}
\hline Parameter & \multicolumn{2}{c}{ Description/Value } \\
\hline & Direct extruder $\quad$ Bowden extruder \\
\cline { 2 - 2 } Bar mount size, L x W x H & $30 \times 13 \times 16 \mathrm{~mm} \quad 100 \times 22 \times 6 \mathrm{~mm}$ \\
Nozzle initial diameter & $0.4 \mathrm{~mm}$ \\
Bar mount material & Aluminium \\
Stepper motor torque & $0.285 \mathrm{~mm}$ \\
Drive gear diameter & $10.8 \mathrm{~mm}$ \\
Filament type & $\mathrm{PLA}, \mathrm{ABS}, \mathrm{SemiFlex}$ \\
Filament diameter & $1.75 \mathrm{~mm}$ \\
Extrusion temperature & $220^{\circ}, 210^{\circ}, 200^{\circ}, 190^{\circ}, 180^{\circ}, 170^{\circ} \mathrm{C}$ \\
Extrusion speed & $50 \mathrm{~mm} / \mathrm{sec}$ \\
Internal nozzle angle & $110^{\circ}$ \\
Chamber temperature & $32^{\circ} \mathrm{C}$ \\
Young's modulus of Aluminium & $70 \mathrm{GPa}$ \\
Young's modulus of PLA & $3.5 \mathrm{GPa}$ \\
PLA melt viscosity & $100 \mathrm{~Pa} \cdot \mathrm{s}$ \\
PLA flow consistency index & $650 \mathrm{~Pa} \cdot \mathrm{s}^{q}$ \\
PLA flow behaviour index & 0.66 \\
\hline
\end{tabular}


Table 2: Comparison of theoretical and experimental results for Direct extruder, PLA filament

\begin{tabular}{|c|c|c|c|c|c|}
\hline \multicolumn{2}{|l|}{ Results } & \multicolumn{2}{|c|}{ Theoretical } & \multicolumn{2}{|c|}{ Experimental } \\
\hline \multirow{3}{*}{ Natural frequency, $\mathrm{Hz}$} & & \multicolumn{2}{|r|}{203.6} & \multicolumn{2}{|r|}{200} \\
\hline & $\begin{array}{l}\text { Mean nozzle } \\
\text { diameter }\end{array}$ & Value & $\begin{array}{c}\text { Percentage } \\
\text { increase (\%) }\end{array}$ & Value & $\begin{array}{l}\text { Percentage } \\
\text { increase (\%) }\end{array}$ \\
\hline & $0.4 \mathrm{~mm}$ & 0.6155 & & 0.6479 & \\
\hline \multirow{5}{*}{$\begin{array}{l}\text { Natural frequency } \\
\text { amplitudes of acceleration } \\
\text { for each nozzle diameter, } \mathrm{m} / \mathrm{s}^{2}\end{array}$} & $0.3969 \mathrm{~mm}$ & 0.6227 & 1.16 & 0.6554 & 1.15 \\
\hline & $0.3901 \mathrm{~mm}$ & 0.6437 & 4.58 & 0.6848 & 5.69 \\
\hline & $0.3813 \mathrm{~mm}$ & 0.7044 & 14.44 & 0.7414 & 14.43 \\
\hline & $0.3659 \mathrm{~mm}$ & 0.8418 & 36.76 & 0.8769 & 35.34 \\
\hline & $0.3454 \mathrm{~mm}$ & 1.0682 & 73.54 & 1.1128 & 71.75 \\
\hline
\end{tabular}


Table 3: Comparison of theoretical and experimental results for Bowden extruder, PLA filament

\begin{tabular}{|c|c|c|c|c|c|}
\hline \multicolumn{2}{|l|}{ Results } & \multicolumn{2}{|c|}{ Theoretical } & \multicolumn{2}{|c|}{ Experimental } \\
\hline \multirow{3}{*}{ Natural frequency, $\mathrm{Hz}$} & & \multicolumn{2}{|r|}{16.5} & \multicolumn{2}{|r|}{16} \\
\hline & $\begin{array}{c}\text { Mean nozzle } \\
\text { diameter }\end{array}$ & Value & $\begin{array}{c}\text { Percentage } \\
\text { increase (\%) }\end{array}$ & Value & $\begin{array}{l}\text { Percentage } \\
\text { increase (\%) }\end{array}$ \\
\hline & $0.35 \mathrm{~mm}$ & 0.2344 & & 0.2520 & \\
\hline \multirow{5}{*}{$\begin{array}{l}\text { Natural frequency } \\
\text { amplitudes of acceleration } \\
\text { for each nozzle diameter, } \mathrm{m} / \mathrm{s}^{2}\end{array}$} & $0.3441 \mathrm{~mm}$ & 0.2503 & 6.78 & 0.2635 & 4.56 \\
\hline & $0.3374 \mathrm{~mm}$ & 0.2632 & 12.28 & 0.2800 & 11.11 \\
\hline & $0.3239 \mathrm{~mm}$ & 0.3233 & 37.92 & 0.3403 & 35.03 \\
\hline & $0.3069 \mathrm{~mm}$ & 0.4458 & 90.18 & 0.4596 & 82.38 \\
\hline & $0.2839 \mathrm{~mm}$ & 0.6370 & 171.75 & 0.6705 & 166.07 \\
\hline
\end{tabular}

